Voix et Images

voixetimages

\title{
Des contes et des nouvelles pour rêver
}

\section{Dominique Garand}

Volume 12, numéro 3 (36), printemps 1987

Yves Beauchemin

URI : https://id.erudit.org/iderudit/200670ar

DOI : https://doi.org/10.7202/200670ar

Aller au sommaire du numéro

\section{Éditeur(s)}

Université du Québec à Montréal

\section{ISSN}

0318-9201 (imprimé)

1705-933X (numérique)

Découvrir la revue

\section{Citer cet article}

Garand, D. (1987). Des contes et des nouvelles pour rêver. Voix et Images, 12(3), 551-555. https://doi.org/10.7202/200670ar d'utilisation que vous pouvez consulter en ligne.

https://apropos.erudit.org/fr/usagers/politique-dutilisation/ 


\section{Des contes et des nouvelles pour rêver}

\section{par Dominique Garand, Université de Montréal}

Il est sans doute trop tôt pour se livrer à une analyse objective des facteurs qui font de la nouvelle, aujourd'hui, un genre de plus en plus pratiqué et valorisé. La "littérature québécoise» avait pourtant, à ses débuts, prisé le texte court, en particulier le conte, qui favorisait le passage dans le corpus écrit d'une riche tradition orale. Il y eut ensuite, dans les vingt premières années du siècle, chez les écrivains régionalistes en particulier, une vogue des récits brefs et des tableaux. Il s'agissait alors de reproduire des scènes vécues, pour fixer à jamais dans la mémoire des gestes et des paysages condamnés à disparaître. Pour plusieurs jeunes écrivains et amateurs, la prose brève constituait un accès privilégié à la littérature, un moyen de parfaire son style tout en obtenant une première audience chez les lecteurs de revues et de journaux. Je crois que l'écrit bref (poème inclus) remplit encore aujourd'hui cette fonction d'apprentissage. Que peu d'écrivains professionnels d'ici fassent de la nouvelle une spécialité, contrairement à ce qui se passe aux États-Unis, cela s'explique bien entendu par l'absence de revues à grand tirage qui rendraient lucrative cette activité spécifique.

On peut enfin, à titre d'hypothèse seulement, relier l'émergence du récit court à l'effritement de la modernité. J'observe en effet, dans ce flot de nouvelles et de contes, un retrait massif des questionnements formels et idéologiques qui avaient cours il n'y a pas si longtemps: on renoue volontiers avec la narration de type traditionnel, avec les effets de fiction les plus classiques, avec une conception esthétisante de la forme, comme si le désir était de faire enfin de "vraies» œuvres d'imagination, de vraies histoires avec personnages et intrigue. L'heure est à l'humilité, à l'apprentissage patient du travail d'écriture. C'est du moins ce qui ressort des trois livres qu'il m'a été donné de lire tout récemment: Aimer ( 10 nouvelles par 10 auteurs québécois), l'Envoleur de chevaux et autres contes par Marie José Thériault et Après l'Éden de Marcel Godin.

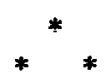

Dans la postface du collectif Aimer ${ }^{1}$, André Carpentier s'attache à décrire l'attitude qu'il souhaiterait voir adopter par les lecteurs des collectifs publiés aux Éditions Quinze. Il insiste entre autres sur la singularité de chaque nouvelle et propose qu'on leur trouve un sens, non en rapport avec les autres nouvelles du collectif, mais dans le cadre de la production globale des écrivains. Cette invitation est d'ailleurs implicitement traduite par les notices bio-bibliographiques qui précèdent chaque texte. Le collectif remplit donc une fonction publicitaire, mais il faut souligner que les auteurs ici rassemblés ne font pas nécessairement partie de l'écurie des Quinze, ce qui honore l'éditeur.

Il est vrai que les dix textes présentent une assez grande variété, dans le style, dans le ton, ou tout simplement dans l'approche du sujet annoncé. Cette diversité rend la critique ardue: sur quels critères faire reposer le juge- 
ment? Il apparaît évident que ces auteurs orientent leur métier d'écrivain selon des stratégies et des exigences différentes, adaptées à des publics divers. Ainsi, les lecteurs qui apprécient les nouvelles réalistes, avec personnages se prêtant à l'identification, auront du plaisir à lire "Éliane et Fred" d'André Berthiaume, même si le dénouement, un peu forcé, laisse perplexe. "Léa et Paul", de Monique Proulx, est par contre assez réussi dans son genre. On assiste aux moment-clés d'un amour, présentés dans un ordre non chronologique, peut-être davantage fidèle aux mécanismes de la mémoire. «Jouez-moi Dumka» de Suzanne Robert exploite le thème du désir mimétique et de l'amour par procuration. Ce thème a déjà inspiré de grandes œuvres (pensons à Dostoïevski), mais ici le résultat n'est pas très puissant, comme si l'auteure ne touchait que du bout des doigts, et raisonnablement, cette passion rivale.

"Le serment de la cuisse», par André Carpentier, expose de son côté le malentendu qui marque l'histoire d'un couple, malentendu dont l'épisode du serment/serrement de la cuisse sera le fondement. "La grande nuit blanche» d'André Major et "Le dernier accrochage" de Diane-Monique Daviau, se présentent également comme des récits linéaires, mais leur ambiance est plus trouble: érotisme et pulsion de mort chez Major, jeux de regards et de miroirs chez Daviau, amours parallèles...

Avec "Docteur l'Indienne», de Victor-Lévy Beaulieu, on entre dans un autre registre. Beaulieu, toujours interpellé par la souillure et le péché, construit une sorte de fable mythique dont les trois principaux actants sont l'Indien dépossédé, le prêtre incarnant la domination blanche et française, et l'enfant-femme (la narratrice) porteuse de vie, d'innocence et de compassion. Plus fondamentalement, l'opposition s'établit entre une divinité "sauvage", la Morue-Mère, source de sexualité libérée, et le «coq gaulois", symbole d'acculturation et de culpabilité.

Marie José Thériault, dans "Mains-Maisons», et Élizabeth Vonarburg dans "La carte du Tendre", traitent toutes deux, mais de façon très éloignée, du thème de la fusion. Chez celle-ci, la fusion s'accomplit dans un hallucinant transfert d'épiderme entre un homme et une femme. Tout cela, bien sûr, à une époque d'extrême sophistication technologique. Chez Marie José Thériault, la fusion est plutôt rêvée, je dirais même idéalisée. C'est toute la rencontre amoureuse qui est métaphorisée par l'image classique des trains qui se croisent, cheminent côte à côte, se fondent ... etc. En filigrane, le fantasme de la gémellité s'installe et Thériault laisse monter les images douces et rassurantes du bonheur amoureux: le cocon, le chaud, Mains-Maisons. En conclusion: Leurs paroles s'emmêlent, on ne sait plus très bien qui parle, qui répond, mais peu importe. Peu importe?

Sans doute parce que je suis un intellectuel, peut-être par affinité inconsciente avec un écrivain de ma génération, j’ai apprécié par dessus tout la contribution de Marc Provencher, "L'agression». La nouvelle est drôle. Le lecteur assiste à la construction de l'histoire, grâce au procédé de la métafiction. Parodie d'un exposé théorique, le récit regorge de trouvailles, sur le climat amoureux de Montréal, sur la mise en scène du désir, sur le conformisme sexuel et les stéréotypes de la représentation fictionnelle. C'est un 
plaisir que d'entendre le narrateur se moquer du moralisme (animiste, dira-til) qui tente de censurer les productions artistiques, sous prétexte qu'elles doivent donner l'exemple du bien: Les scènes de violence, on le sait, transforment automatiquement les plus placides assistances en hordes de bêtes féroces prêtes à se répandre dans nos rues (p. 116). Les formules abondent et la distanciation que prend le narrateur à l'égard de son histoire ne nuit pas au plaisir de la lecture. Bref, l'auteur, qui à l'instar des héros stendhaliens dit ne s'être intéressé que sporadiquement à l'amour, et presque toujours par calcul, revendique avec humour le droit au malheur (cf. l'épigraphe de Huxley), ce qui est une autre façon de réclamer le droit au désir. Et conformément aux lois du genre, la nouvelle se termine bel et bien par une chute inattendue.

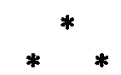

La critique a dit beaucoup de bien de l'Envoleur de chevaux ${ }^{2}$. La présentation matérielle du livre attire le regard; un univers onirique particulier semble se dégager du recueil. Ai-je été déçu? Un peu, mais je ne sais trop comment l'expliquer: par une faiblesse d'ensemble du livre ou par la tournure de mon imaginaire, qui n'aurait pas réussi à rencontrer celui de l'auteure?

Ce sont vingt contes d'inégale longueur, qui font un peu penser aux histoires illustrées d'Épinal. Ils portent tous une certaine charge symbolique. Le lecteur qui a gardé de l'enfance la nostalgie des fables énigmatiques sera servi. Retrouver le temps perdu? L'imaginaire exotique? Le merveilleux symbolique? Le désir sans objet? La pulsion fantasmée? Le mystère originel? Le tout sur fond allégorique de scène primitive et vaguement incestueuse? Voilà. Dans le genre, c'est bien fait. Je pense que ce livre a son importance, car il en existe peu de semblables dans la production québécoise. Il se situe loin des esthétiques dominantes et peut satisfaire le public des adolescents (bourgeois) autant que celui des adultes.

Le métier de Marie José Thériault ne fait aucun doute. Le vocabulaire qu'elle utilise est somptueux, sinon recherché, et sa culture diversifiée. On trouve plusieurs références mythiques ou historiques (Rameau d'or, Alcyon, Lucrèce, Messaline...) et quelques "remakes" de textes fonduateürs ("Genèse", "Mille et une nuits»). L'écriture, lente, suggestive, est ritualisée par un retour marqué au style canonique des contes séculaires, mais aussi par le discours des personnages, tous un peu figés (on les imagine avec de grands yeux rêveurs, tendus vers l'horizon), intemporels, et qui se vouvoient continuellement, comme dans les romans de Marguerite Duras. Parfois, on sent trop le travail de l'auteure, on devine plus qu'on éprouve les émotions ou les sensations qu'elle veut nous procurer. Peut-être parce que ses effets sont un peu trop codés. Par exemple, je ne ressens pas vraiment la cruauté mise en scène dans "Anna Méloé» et dans «Messaline»: l'écriture ne réussit pas à me faire oublier que je lis une œuvre littéraire.

L'un des aspects les plus intéressants du recueil concerne le rapport entre l'homme et la femme. Dans ce rapport, l'homme est souvent le jouet de l'aimée - il est fou, soumis, possédé; elle, toute-puissante, parfois tyrannique, insatiable. Il est surtout intéressant de constater que dans la plupart des 
contes, le rapport n'a pas vraiment lieu, sinon comme un non-rapport. À cet égard, "L'impossible train d'Anvers» est un conte exemplaire: entre Lui et Elle, il y a tout l'espace du fantasme... et le regard du lecteur, "incarné» par les voyeurs du train. On se cherche, on s'attend, mais on se parle peu et quand cela arrive, c'est de façon ritualisée, avec des poses hiératiques; de même, la rencontre amoureuse est presque toujours furtive, ou différée: les personnages sont littéralement visités par l'amour, beaucoup plus qu'ils ne le rencontrent. Marie José Thériault touche ici une vérité du rapport sexuel qui est, comme l'affirmait naguère Lacan, impossible. Mais il n'est pas sûr que l'auteure veuille renoncer au fantasme de fusion avec l'Autre (religieusement convié). Comme l'atteste la petite nouvelle parue dans Aimer, cet Autre revêt souvent la figure du Même, du Jumeau quasi indistinct du Moi. La jouissance, dans ces contes, fonctionne à la répétition; l'écriture même est tendue vers le modèle du Texte sacré. Ce n'est pas pour rien que le recueil se clôt sur l'archétype des Mille et une nuits: points de suspension, désir d'une nouvelle histoire, au cas où...

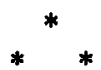

Après l'Éden ${ }^{3}$ n'est certainement pas un sommet dans l'œuvre de Marcel Godin. On dirait qu'il a voulu se payer une petite vacance, un livre facile. Pourtant, il sait à l'occasion se montrer bon conteur, mais les huit «nouvelles » qu'il nous présente n'ont, dans l'ensemble, rien de particulièrement intéressant. Premièrement, ce ne sont pas des nouvelles: la première partie, "L'endroit ", est composée de quatre tableaux, ou évocations, tandis que la seconde partie, "L'envers», fait place à quatre textes ésotériques, symboliques, beaucoup plus proches du poème en prose que de la nouvelle.

Mais n'insistons pas sur cette question, somme toute assez superficielle, des genres. Quel est le propos général du livre? Le prière d'insérer nous donne une piste: "l'endroit" et "l'envers" seraient les deux facettes de notre réalité d' "après l'Éden", la vécue et la rêvée. Dans un court paragraphe, on voit défiler une série d'oppositions binaires: perception/invention, ombre/ lumière, souvenir/imagination, haut/bas, amour/haine, vie/mort, ciel/enfer, réalisme/surréalisme. La structure du livre respecte cette donnée: la première partie laisse place à quatre souvenirs associés aux saisons. L'auteur s'y montre tendre et moraliste (au sens large). Dans «Le printemps» et «L'automne», il montre que l'on peut jouir de la contradiction, soit que la transgression conduise à la connaissance intime de la vie, soit que l'érotisme s'allie au meurtre de façon troublante.

La deuxième partie explore davantage le territoire symbolique. À partir des points cardinaux, on suit l'élaboration de quatre royaumes distincts, qui peuvent être vus également comme des "territoires de l'âme", des pulsions contradictoires présentes dans la psychè. Mais tout cela est assez ésotérique et mises à part certaines pistes de lecture (Nord-blanc-froid-ordre-obsession, Sud-noir-fête-luxuriance, Ouest-rouge-sang-violence, etc.), l'ensemble reste un peu inaccompli. 
En résumé, les trois livres présentés ci-dessus s'en tiennent à une forme et à un contenu assez conservateurs. Dans Aimer, peu d'auteurs ont pris de risque; on reste gentil, rassurant, pudique et l'emportement, la douleur, la jouissance, la transgression se font rares. Certains touchent une véritable expressivité qu'ils sauront exploiter plus intensément, espérons-le. La nouvelle est un genre qui, malgré (ou grâce à?) ses règles strictes, permet de multiples jeux formels. Si une certaine émulation est en train de naître parmi ceux qui la pratiquent, on ne peut que s'en réjouir. L'écriture d'une Marie José Thériault témoigne d'une recherche d'unité et de qualité: on sent chez elle un projet. Le mythe, il est vrai, transporte un très riche trésor de signification; je doute toutefois que la mythification et l'esthétisation permettent à l'écrivain de franchir le miroir. Mais, au fait, qui tente de traverser le miroir? La plupart des écrivains n'aspirent qu'à s'y mirer, avec fascination.

1. Aimer, 10 nouvelles par 10 auteurs québécois (équipe dirigée par André Carpentier), Montréal, Les Quinze, 1986, $192 \mathrm{p}$.

2. Marie José Thériault, l'Envoleur de chevqux et autres contes, Montréal, Boréal, 1986, $180 \mathrm{p}$.

3. Marcel Godin, Après l'Éden, Montréal, l'Hexagone, 1986, 102 p.

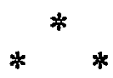

